\title{
A canonical basis for Garsia-Procesi modules
}

\author{
Jonah Blasiak ${ }^{1 \rrbracket}$ \\ ${ }^{1}$ The University of Chicago, Department of Computer Science, 1100 East 58th Street, Chicago, IL 60637, USA
}

\begin{abstract}
We identify a subalgebra $\widehat{\mathscr{H}}_{n}^{+}$of the extended affine Hecke algebra $\widehat{\mathscr{H}}_{n}$ of type $A$. The subalgebra $\widehat{\mathscr{H}}_{n}^{+}$ is a $u$-analogue of the monoid algebra of $\mathcal{S}_{n} \ltimes \mathbb{Z}_{\geq 0}^{n}$ and inherits a canonical basis from that of $\widehat{\mathscr{H}_{n}}$. We show that its left cells are naturally labeled by tableaux filled with positive integer entries having distinct residues mod $n$, which we term positive affine tableaux (PAT).

We then exhibit a cellular subquotient $\mathscr{R}_{1^{n}}$ of $\widehat{\mathscr{H}}_{n}^{+}$that is a $u$-analogue of the ring of coinvariants $\mathbb{C}\left[y_{1}, \ldots, y_{n}\right] /\left(e_{1}, \ldots, e_{n}\right)$ with left cells labeled by PAT that are essentially standard Young tableaux with cocharge labels. Multiplying canonical basis elements by a certain element $\pi \in \widehat{\mathscr{H}}_{n}^{+}$corresponds to rotations of words, and on cells corresponds to cocyclage. We further show that $\mathscr{R}_{1^{n}}$ has cellular quotients $\mathscr{R}_{\lambda}$ that are $u$-analogues of the Garsia-Procesi modules $R_{\lambda}$ with left cells labeled by (a PAT version of) the $\lambda$-catabolizable tableaux.
\end{abstract}

Résumé. On définit une sous-algèbre $\widehat{\mathscr{H}}_{n}^{+}$de l'extension affine de l'algèbre de Hecke $\widehat{\mathscr{H}}_{n}$ de type $A$. La sousalgèbre $\widehat{\mathscr{P}}_{n}^{+}$est $u$-analogue à l'algèbre monoïde de $\mathcal{S}_{n} \ltimes \mathbb{Z}_{>0}^{n}$ et hérite d'une base canonique de $\widehat{\mathscr{P}}_{n}$. On montre que ses cellules gauches sont naturellement classées par des tableaux remplis d'entiers naturels ayant chacun des restes différents modulo $n$, que l'on nomme Positive Affine Tableaux (PAT).

On montre ensuite qu'un sous-quotient cellulaire $\mathscr{R}_{1}^{n}$ de $\widehat{\mathscr{H}}_{n}^{+}$est une $u$-analogue de l'anneau des co-invariants $\mathbb{C}\left[y_{1}, \ldots, y_{n}\right] /\left(e_{1}, \ldots, e_{n}\right)$ avec des cellules gauches classées PAT qui sont essentiellement des tableaux de Young standards avec des labels cochargés. Multiplier les éléments de la base canonique par un certain élément $\pi \in \widehat{\mathscr{H}}_{n}^{+}$ correspond à des rotations de mots, et par rapport aux cellules cela correspond à un cocyclage. Plus loin, on montre que $\mathscr{R}_{1}{ }^{n}$ a pour quotients cellulaires $\mathscr{R}_{\lambda}$ qui sont $u$ - analogues aux modules de Garsia-Procesi $R_{\lambda}$ avec des cellules gauches définies par (une version PAT) des tableaux $\lambda$-catabolisable.

Keywords: Garsia-Procesi modules, affine Hecke algebra, canonical basis, symmetric group, $k$-atoms

\section{Introduction}

It is well-known that the ring of coinvariants $R_{1^{n}}=\mathbb{C}\left[y_{1}, \ldots, y_{n}\right] /\left(e_{1}, \ldots, e_{n}\right)$, thought of as a $\mathbb{C S}_{n^{-}}$ module with $\mathcal{S}_{n}$ acting by permuting the variables, is a graded version of the regular representation. However, how a decomposition of this module into irreducibles is compatible with multiplication by the $y_{i}$ remains a mystery.

A precise question one can ask along these lines goes as follows. Let $E \subseteq R_{d}$ be an $\mathcal{S}_{n}$-irreducible, where $R_{d}$ is the $d$-th graded part of the polynomial ring $R=\mathbb{C}\left[y_{1}, \ldots, y_{n}\right]$. Suppose that the isotypic

$\dagger$ Is currently an NSF postdoctoral fellow. This research was partially conducted during the period the author was employed by the Clay Mathematics Institute as a Liftoff Fellow.

1365-8050 @ 2010 Discrete Mathematics and Theoretical Computer Science (DMTCS), Nancy, France 
component of $R_{d}$ containing $E$ is $E$ itself. Then define $I \subseteq R$ to be the sum of all homogeneous ideals $J \subseteq R$ that are left stable under the $\mathcal{S}_{n}$-action and satisfy $J \cap E=0$. The quotient $R / I$ contains $E$ as the unique $\mathcal{S}_{n}$-irreducible of top degree $d$. It is natural to ask

What is the graded character of $R / I$ ?

The most familiar examples of such quotients are the Garsia-Procesi modules $R_{\lambda}$ (see [5]), which correspond to the case that $E$ is of shape $\lambda$ and $d=n(\lambda)=\sum_{i}(i-1) \lambda_{i}$; refer to this representation $E \subseteq R_{n(\lambda)}$ as the Garnir representation of shape $\lambda$ or, more briefly, $G_{\lambda}$. Combining the work of HottaSpringer (see [6]) and Lascoux [10] (see also [15]) gives the Frobenius series

$$
\mathscr{F} R_{\lambda}(t)=\sum_{\substack{T \in S Y T \\ \operatorname{ctype}(T) \unrhd \lambda}} t^{\operatorname{cocharge}(T)} s_{\mathrm{sh}(T)},
$$

where ctype $(T)$ is the catabolizability of $T$ (see $\$ 4$.

Though this interpretation of the character of $R_{\lambda}$ has been known for some time, the only proofs were difficult and indirect. One of the goals of this research, towards which we have been partially successful, was to give a more transparent explanation of the appearance of catabolism in the combinatorics of the coinvariants.

More recent work suggests that there are other combinatorial mysteries hiding in the ring of coinvariants. We strongly suspect that modules with graded characters corresponding to the $k$-atoms of Lascoux, Lapointe, and Morse [9] and a generalization of $k$-atoms due to Li-Chung Chen [4] sit inside the coinvariants as subquotients. It is also natural to conjecture that the generalization of catabolism due to Shimozono and Weyman [15] gives a combinatorial description of certain subquotients of the coinvariants which are graded versions of induction products of $\mathcal{S}_{n}$-irreducibles.

This paper describes an approach to these problems using canonical bases, which has so far been quite successful and will hopefully help solve some of the difficult conjectures in this area. After briefly reviewing Weyl groups, Hecke algebras, and cells (\$2), we introduce the central algebraic object of our work, a subalgebra $\widehat{\mathscr{H}}^{+}$of the extended affine Hecke algebra which is a $u$-analogue of the monoid algebra of $\mathcal{S}_{n} \ltimes \mathbb{Z}_{\geq 0}^{n}$. In $\sqrt[3]{3}$, we establish some basic properties of this subalgebra and describe its left cells. It turns out that these cells are naturally labeled by tableaux filled with positive integer entries having distinct residues $\bmod n$, which we term positive affine tableaux (PAT). Our investigations have convinced us that these are excellent combinatorial objects for describing graded $\mathcal{S}_{n}$-modules.

After some preparatory combinatorics in $\S 4$, we go on to show in $\S 5$ that $\widehat{\mathscr{H}}^{+}$has a cellular quotient $\mathscr{R}_{1^{n}}$ that is a $u$-analogue of $R_{1^{n}}$. The module $\mathscr{R}_{1^{n}}$ has a canonical basis labeled by affine words that are essentially standard words with cocharge labels, with left cells labeled by PAT that are essentially standard tableaux with cocharge labels. Multiplying canonical basis elements by a certain element $\pi \in \widehat{\mathscr{H}}^{+}$ corresponds to rotations of words, and on left cells corresponds to cocyclage.

In this cellular picture of the coinvariants, $G_{\lambda}$ corresponds to a left cell of $\mathscr{R}_{1^{n}}$ labeled by a PAT of shape $\lambda$, termed the Garnir tableau of shape $\lambda$, again denoted $G_{\lambda}$. In $\$ 6$, we identify $u$-analogues $\mathscr{R}_{\lambda}$ of the $R_{\lambda}$ and show that $\mathscr{R}_{\lambda}$ is cellular and its left cells are labeled by (a PAT version of) the $\lambda$-catabolizable tableaux.

Detailed proofs as well as conjectures relating cellular subquotients of $\widehat{\mathscr{H}}^{+}$to $k$-atoms and Chen's atoms and conjectures describing cellular subquotients of $\widehat{\mathscr{H}}^{+}$outside of $\mathscr{R}_{1}$ are presented in a full version of this extended abstract [1]. 


\section{Hecke algebras and cells}

We begin by briefly reviewing Weyl groups and Hecke algebras, referring the reader to [7] for a thorough treatment.

Let $W_{f}, W_{a}, W_{e}, Y, Y_{+}$be the finite Weyl group, affine Weyl group, extended affine Weyl group, weight lattice, and dominant weights associated to the root system specifying the algebraic group $G L_{n}(\mathbb{C})$ (see [7]). The finite Weyl group $W_{f}$ is the symmetric group $\mathcal{S}_{n}$. It acts on the weight lattice $Y=\mathbb{Z}^{n}=$ $\left\langle\epsilon_{1}, \ldots, \epsilon_{n}\right\rangle$ by permuting coordinates.

Let $S=\left\{s_{1}, \ldots, s_{n-1}\right\}$ be the simple reflections of $W_{f}$ and $K=\left\{s_{0}, \ldots, s_{n-1}\right\}$ be those of $W_{a}$ and $W_{e}$. The pairs $\left(W_{f}, S\right)$ and $\left(W_{a}, K\right)$ are Coxeter groups, and $\left(W_{e}, K\right)$ is an extended Coxeter group. The length function $\ell$ and partial order $\leq$ on $W_{a}$ extend to $W_{e}=\Pi \ltimes W_{a}: \ell(\pi v)=\ell(v)$, and $\pi v \leq \pi^{\prime} v^{\prime}$ if and only if $\pi=\pi^{\prime}$ and $v \leq v^{\prime}$, where $\pi, \pi^{\prime} \in \Pi, v, v^{\prime} \in W$. For any $J \subseteq K$, the parabolic subgroup $W_{e J}=W_{a J}$ is the subgroup of $W_{e}$ generated by $J$. Each left (resp. right) coset $w W_{e J}$ (resp. $W_{e J} w$ ) of $W_{e J}$ contains a unique element of minimal length called a minimal coset representative. The set of all such elements is denoted $W_{e}^{J}$ (resp. ${ }^{J} W_{e}$ ).

We will make use of three descriptions of $W_{e}$. First, $W_{e}=Y \rtimes W_{f}$; elements of $Y \subseteq W_{e}$ will be denoted by the multiplicative notation $y^{\lambda}, \lambda \in Y$ and $y_{i}:=y^{\epsilon_{i}}$. Second, $W_{e}=\Pi \ltimes W_{a}$, where $\Pi \cong \mathbb{Z}$; the element $\pi=y_{1} s_{1} s_{2} \ldots s_{n-1}$ is a generator of $\Pi$. This satisfies the relation $\pi s_{i}=s_{i+1} \pi$, where, here and from now on, the subscripts of the $s_{i}$ are taken $\bmod n$.

The third description of $W_{e}$, due to Lusztig, identifies it with the group of permutations $w: \mathbb{Z} \rightarrow \mathbb{Z}$ satisfying $w(i+n)=w(i)+n$ and $\sum_{i=1}^{n}(w(i)-i) \equiv 0 \bmod n$. The identification takes $s_{i}$ to the permutation transposing $i+k n$ and $i+1+k n$ for all $k \in \mathbb{Z}$, and takes $\pi$ to the permutation $k \mapsto k+1$ for all $k \in \mathbb{Z}$. We take the convention of specifying the permutation of an element $w \in W_{e}$ by the word

$$
n+1-w^{-1}(1) n+1-w^{-1}(2) \ldots n+1-w^{-1}(n) .
$$

We refer to this as the affine word or word of $w$, and it will be written as $w_{1} w_{2} \cdots w_{n}$; this is understood to be part of an infinite word so that $w_{i}=\hat{i}-i+w_{\hat{i}}$, where $\hat{\imath}: \mathbb{Z} \rightarrow[n]$ is the map sending an integer $i$ to the integer in $[n]$ it is congruent to $\bmod n$. For example, if $n=4$ and $w=\pi^{2} s_{2} s_{0} s_{1}$, then the word of $w$

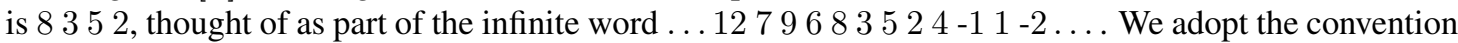
of writing $a b$ in place of $n a+b(a, b \in \mathbb{Z})$. With this convention, the word of $w$ above is written 143112 .

Let $A=\mathbb{Z}\left[u, u^{-1}\right]$ be the ring of Laurent polynomials in the indeterminate $u$. Let $\mathscr{H}(W)$ be the Hecke algebra of the (extended) Coxeter group $W$ over the ground ring $A$ with standard basis $\left\{T_{w}: w \in W\right\}$. Set $\mathscr{H}=\mathscr{H}\left(W_{f}\right), \widehat{\mathscr{H}}=\mathscr{H}\left(W_{e}\right)$, which will sometimes be decorated with a subscript $n$ to emphasize that they correspond to type $A_{n-1}$ or $\tilde{A}_{n-1}$. The Hecke algebra of an extended Coxeter group has the same relations as the usual Hecke algebra using the length function defined above.

Corresponding to the description $Y \rtimes W_{f}$ of $W_{e}$, there is a presentation of $\widehat{\mathscr{\mathscr { H }}}$ due to Bernstein. For any $\lambda \in Y$ there exist $\mu, \nu \in Y_{+}$such that $\lambda=\mu-\nu$. Define $Y^{\lambda}:=T_{y^{\mu}}\left(T_{y^{\nu}}\right)^{-1}$, which is independent of the choice of $\mu$ and $\nu$. The algebra $\widehat{\mathscr{H}}$ has $A$-basis

$$
\left\{Y^{\lambda} T_{w}: w \in W_{f}, \lambda \in Y\right\}
$$

and is equal to the $A$-algebra generated by the $Y_{i}$ and $T_{s_{i}}$ with relations that are fairly simple to describe.

The canonical basis or Kazhdan-Lusztig basis of $\mathscr{H}(W)[8]$ is an $A$-basis for $\mathscr{H}(W)$, denoted $\left\{C_{w}^{\prime}\right.$ : $w \in W\}$, having nice properties for the action of the Hecke algebra on itself. This action is nice because 
certain subsets of the canonical basis called cells give rise to representations that are often irreducible. Cells can be defined for any $\mathscr{H}(W)$-module $E$ with a distinguished basis $\Gamma$ : first, the preorder $\leq_{\Gamma}$ (also denoted $\leq_{E}$ ) on the set $\Gamma$ is that generated by the relations/edges

$$
\delta \overleftarrow{\Gamma}_{\gamma} \begin{gathered}
\text { if there is an } h \in \mathscr{H}(W) \text { such that } \delta \text { appears with non-zero } \\
\text { coefficient in the expansion of } h \gamma \text { in the basis } \Gamma .
\end{gathered}
$$

The left cells of $\Gamma$ (or of $E$, if $\Gamma \subseteq E$ is understood) are then the equivalence classes of $\leq_{\Gamma}$. The preorder $\leq_{\Gamma}$ gives rise to a partial order on left cells, also denoted $\leq_{\Gamma}$. A cellular subquotient of $E$ is a subset $\Lambda$ of $\Gamma$ such that there does not exist $\gamma \in \Gamma \backslash \Lambda$ and $\delta, \delta^{\prime} \in \Lambda$ satisfying $\delta \leq_{\Gamma} \gamma \leq_{\Gamma} \delta^{\prime}$. A cellular subquotient of $E$ is necessarily a union of left cells and gives rise to a subquotient of $E$. We are most interested in the case where $\Gamma$ is a $W$-graph as defined in [8].

\section{The positive part of $\widehat{\mathscr{H}}$}

Here we introduce a subalgebra $\widehat{\mathscr{H}}^{+}$of $\widehat{\mathscr{H}}$ and positive affine tableaux (PAT), which label left cells of $\widehat{\mathscr{H}}^{+}$. These play a crucial role in our goal of relating subquotients of $R$ to tableau combinatorics.

The subset $Y^{+}:=\mathbb{Z}_{\geq 0}^{n}$ of the weight lattice $Y$ is left stable under the action of the Weyl group $W_{f}$. Thus $Y^{+} \rtimes W_{f}$ is a submonoid of $W_{e}$. We remark that this only works in type $A$, and this is the main barrier preventing the results of this paper to be generalized to other types.

Proposition-Definition 3.1 The positive part of $W_{e}$, denoted $W_{e}^{+}$, has the following three equivalent descriptions:

(1) $Y^{+} \rtimes W_{f}$,

(2) The submonoid of $W_{e}$ generated by $\pi$ and $W_{f}$,

(3) $\left\{w \in W_{e}: w_{i}>0\right.$ for all $\left.i \in[n]\right\}$.

The inclusion of monoids $W_{e}^{+} \subseteq W_{e}$ gives rise to an inclusion of algebras $\widehat{\mathscr{H}}^{+} \subseteq \widehat{\mathscr{H}}$ :

Proposition-Definition 3.2 The subalgebra $\widehat{\mathscr{H}}^{+}$of $\widehat{\mathscr{H}}$ has the following four equivalent descriptions:

(i) $A\left\{Y^{\lambda} T_{w}: \lambda \in Y^{+}, w \in W_{f}\right\}$,

(ii) $A\left\{T_{w}: w \in W_{e}^{+}\right\}$,

(iii) $A\left\{C_{w}^{\prime}: w \in W_{e}^{+}\right\}$,

(iv) the subalgebra of $\widehat{\mathscr{H}}$ generated by $\pi$ and $\mathscr{H}$.

Write $\leq_{\widehat{\mathscr{H}}^{+}}$for the preorder on the canonical basis of $\widehat{\mathscr{H}}^{+}$coming from considering $\widehat{\mathscr{H}}^{+}$as a left $\widehat{\mathscr{H}}^{+}$-module. We say that this canonical basis is the $W_{e}^{+}$-graph $\Gamma_{W_{e}^{+}}$. The preorder $\leq \widehat{\mathscr{H}}^{+}$is difficult to compute, but there are two kinds of easy edges: the edges $C_{\pi w}^{\prime} \leq \widehat{\mathscr{H}}^{+} C_{w}^{\prime}$, which we refer to as corotationedges; the corresponding edges between cells are cocyclage-edges (we will soon see that cocyclage-edges are a generalization of cocyclage for standard Young tableaux). The edges $C_{s w}^{\prime} \leq_{\widehat{\mathscr{H}}^{+}} C_{w}^{\prime}$ if $s w>w$ and $s \in S$ are ascent-edges.

The work of Kazhdan and Lusztig [8] shows that the left cells of $\mathscr{H}$ are in bijection with the set of SYT and the left cell containing $C_{w}^{\prime}$ corresponds to the insertion tableau of $w$ under this bijection (keep in mind our unusual convention from $\$ 2$ for the word of $w$ ). The left cell containing those $C_{w}^{\prime}$ such that $w$ has insertion tableau $P$ is the left cell labeled by $P$, denoted $\Gamma_{P}$. 
Definition 3.3 A positive affine tableau (PAT) of size $n$ is a semistandard Young tableau filled with positive integer entries that have distinct residues mod $n$.

For $w \in W_{e}$, the word $w_{1} w_{2} \cdots w_{n}$ may be inserted into a tableau, and the result is a tableau, denoted $P(w)$. It is a positive affine tableau exactly when $w \in W_{e}^{+}$. Let $Q$ be a positive affine tableau and let $Q_{S}$ be the standard tableau obtained from $Q$ by replacing its entries with the numbers $1, \ldots, n$ so that the relative order of the entries in $Q$ and $Q_{S}$ agree. The set of $w \in W_{e}$ inserting to $Q$ is $\{v x: v \in$ $W_{f}$ and $\left.P(v)=Q_{S}\right\}$, where the word of $x$ is obtained from $Q$ by sorting its entries in decreasing order. For any $x \in{ }^{S} W_{e}$, define

$$
\Gamma_{Q}:=\left\{C_{v x}^{\prime}: v \in W_{f}, P(v)=Q_{S}\right\}=\left\{C_{w}^{\prime}: w \in W_{e}, P(w)=Q\right\} .
$$

By the following result, $\Gamma_{Q}$ is a left cell of $\Gamma_{W_{e}^{+}}$, which we refer to as the left cell labeled by $Q$. The following is an easy consequence of results of Roichman on restricting $W$-graphs that originated in the work of Barbasch and Vogan on primitive ideals (see [14]).

Proposition 3.4 For any $x \in{ }^{S} W_{e}^{+}$, the set $\left\{C_{w x}^{\prime}: w \in W_{f}\right\}$ is a cellular subquotient of $\widehat{\mathscr{H}}^{+}$. This set, restricted to be a $W_{f}$-graph, is isomorphic to the $W_{f}$-graph on $\mathscr{H}$. In particular,

$$
\Gamma_{W_{e}^{+}}=\bigsqcup_{Q \in P A T} \Gamma_{Q}
$$

is the decomposition of $\Gamma_{W_{e}^{+}}$into left cells.

\section{Cocyclage and catabolism}

Before going deeper into the study of the canonical basis of $\widehat{\mathscr{H}}^{+}$, we introduce combinatorics originating in [10, 11] (see also [15]) that will be used to describe cellular subquotients of $\widehat{\mathscr{H}}^{+}$.

The cocharge labeling of a word $v$, denoted $v^{\mathrm{cc}}$, is a (non-standard) word of the same length as $v$, and its numbers are thought of as labels of the numbers of $v$. It is obtained from $v$ by reading the numbers of $v$ in increasing order, labeling the 1 of $v$ with a 0 , and if the $i$ of $v$ is labeled by $k$, then labeling the $i+1$ of $v$ with a $k$ (resp. $k+1$ ) if the $i+1$ in $v$ appears to the right (resp. left) of the $i$ in $v$. For example, the cocharge labeling of 614352 is 302120 ; also see Example 5.3 Define the cocharge labeling $T^{\mathrm{cc}}$ of a tableau $T$ to be the insertion tableau of $v^{\text {cc }}$ for any (every) $v$ inserting to inserting to $T$.

The sum of the numbers in the cocharge labeling of a standard word $v$ (resp. standard tableau $T$ ) is the cocharge of $v$ (resp. $T$ ) or cocharge $(v)$ (resp. cocharge $(T)$ ).

For a word $w$ and number $a \neq 1, a w$ is a corotation of $w a$. There is a cocyclage from the tableau $T$ to the tableau $T^{\prime}$, written $T \stackrel{\mathrm{cc}}{\longrightarrow} T^{\prime}$, if there exist words $u, v$ such that $v$ is the corotation of $u$ and $P(u)=T$ and $P(v)=T^{\prime}$. Rephrasing this condition solely in terms of tableaux, $T \stackrel{\text { cc }}{\rightarrow} T^{\prime}$ if there exists a corner square $(r, c)$ of $T$ and uninserting the square $(r, c)$ from $T$ yields a tableau $Q$ and number $a$ such that $T^{\prime}$ is the result of column-inserting $a$ into $Q$.

The cocyclage poset $\mathrm{CCP}(\mathrm{SYT})$ is the poset on the set of SYT generated by the relation $\stackrel{\mathrm{cc}}{\longrightarrow}$. Similarly, define $\mathrm{CCP}(P A T)$ to be the poset on the set of PAT generated by cocyclage-edges. The covering relations of $\mathrm{CCP}(\mathrm{SYT})$ (resp. $\mathrm{CCP}(P A T)$ ) are exactly cocyclages (resp. cocyclage-edges). We consider the covering relation $T \stackrel{\text { cc }}{\longrightarrow} T^{\prime}$ to be colored by the following additional datum: the set of outer corners of $T$ that result in a cocyclage to $T^{\prime}$. Note that this set can only have more than one element if $\operatorname{sh}(T)=\operatorname{sh}\left(T^{\prime}\right)$. 
Catabolizability of standard tableaux is a subtle combinatorial statistic, which we will not define in the usual way here. In [3], we show that the catabolizability of a standard tableau $T$, denoted ctype $(T)$, can be computed from any word $v$ inserting to $T$ using the following catabolism insertion algorithm.

Algorithm 4.1 (Catabolism insertion) Let $f$ be the function below, which takes a pair consisting of a (non-standard) word and a partition to another such pair. Let $x=y a, y$ a word and a $a$ number.

$$
f(x, \nu)= \begin{cases}\left(y, \nu+\epsilon_{a+1}\right) & \text { if } \nu+\epsilon_{a+1} \text { is a partition, } \\ (a+1 y, \nu) & \text { otherwise. }\end{cases}
$$

Given the input standard word $v$, first determine the cocharge labeling z of $v$. Next, apply $f$ to $(z, \emptyset)$ repeatedly until the word of the pair is empty. Output the partition of this final pair.

Example 4.2 The sequence of word-partition pairs produced by the algorithm run on $v=168429573$ is (reading from left to right and then top to bottom)

$\begin{array}{lllll}(023103120, \emptyset) & (02310312,(1)) & (30231031,(1)) & (3023103,(1,1)) & (4302310,(1,1)) \\ (430231,(2,1)) & (43023,(2,2)) & (44302,(2,2)) & (4430,(2,2,1)) & (443,(3,2,1)) \\ (44,(3,2,1,1)) & (4,(3,2,1,1,1)) & (5,(3,2,1,1,1)) & (\emptyset,(3,2,1,1,1,1)) & \end{array}$

\section{A $W_{e}^{+}$-graph version of the coinvariants}

We exhibit a cellular subquotient $\mathscr{R}_{1^{n}}$ of $\widehat{\mathscr{H}}^{+}$which is a $W_{e}^{+}$-graph version of the ring of coinvariants $R_{1^{n}}$. Under a natural identification of the left cells of $\mathscr{R}_{1^{n}}$ with SYT, the subposet of $\leq_{\mathscr{R}_{1}{ }^{n}}$ consisting of the cocyclage-edges is exactly the cocyclage poset on SYT.

There are two important theorems that give the canonical basis of $\widehat{\mathscr{H}}$ a more explicit description.

The dominant weights $Y_{+}$are weakly decreasing $n$-tuples of integers; put $Y_{+}^{+}=Y^{+} \cap Y_{+}$. As is customary, let $w_{0}$ denote the longest element of $W_{f}$. If $\lambda \in Y_{+}$, then $w_{0} y^{\lambda}$ is maximal in its double coset $W_{f} y^{\lambda} W_{f}$. For $\lambda \in Y_{+}^{+}$, let $s_{\lambda}(Y) \in \widehat{\mathscr{H}}$ denote the Schur function of shape $\lambda$ in the Bernstein generators $Y_{i}$.

Theorem 5.1 (Lusztig [12, Proposition 8.6]) For any $\lambda \in Y_{+}^{+}$, the canonical basis element $C_{w_{0} y^{\lambda}}^{\prime}$ can be expressed in terms of the Bernstein generators as

$$
C_{w_{0} y^{\lambda}}^{\prime}=s_{\lambda}(Y) C_{w_{0}}^{\prime}=C_{w_{0}}^{\prime} s_{\lambda}(Y)
$$

Let $v$ be an element of $W_{f}$, thought of as a standard word. Thinking of the cocharge labeling $v^{\mathrm{cc}}$ as an element of $Y^{+}$, let $D \subset Y^{+}$denote the set of cocharge labelings, which is in bijection with $W_{f}$. The set $\left\{y^{\beta}: \beta \in D\right\}$ are the descent monomials. Next, put

$$
\begin{aligned}
D^{S} & :=\left\{y^{\beta} v: \beta \in D \text { and } v \in W_{f} \text { such that } y^{\beta} v \text { is minimal in } y^{\beta} W_{f}\right\}, \\
D^{S} w_{0} & :=\left\{y^{\beta} v: \beta \in D \text { and } v \in W_{f} \text { such that } y^{\beta} v \text { is maximal in } y^{\beta} W_{f}\right\} .
\end{aligned}
$$

The set $D^{S} w_{0}$ will index a canonical basis of the coinvariants. 
Proposition 5.2 There is a bijection $W_{f} \rightarrow D^{S} w_{0}, v \mapsto w$, defined by setting the word of $w$ to be $w_{i}=n v_{i}^{c c}+v_{i}$. Its inverse has the two descriptions

$$
\begin{aligned}
w_{S} & \hookleftarrow w \\
\hat{w}_{1} \hat{w}_{2} \ldots \hat{w}_{n} & \hookleftarrow w
\end{aligned}
$$

where $\hat{w}_{i}$ is the residue of $w_{i}$ as defined in $\$ 2$ and $w_{S}$ is the standard word such that the relative order of the entries in $w_{S}$ and $w$ agree.

Example 5.3 For the $v \in \mathcal{S}_{9}$ given by its word below, the corresponding $v^{c c}$ and $w$ follow.

$$
\begin{aligned}
& v=\begin{array}{llllllllll}
v & 6 & 8 & 4 & 2 & 9 & 5 & 7 & 3,
\end{array} \\
& v^{c c}=\begin{array}{lllllllll}
0 & 2 & 3 & 1 & 0 & 3 & 1 & 2 & 0,
\end{array} \\
& w=n v^{c c}+v=\begin{array}{lllllllll}
1 & 26 & 38 & 14 & 2 & 39 & 15 & 27 & 3 .
\end{array}
\end{aligned}
$$

As preparation for the next theorem, we have a proposition giving the factorization of any $w \in W_{e}^{+}$ with $w$ maximal in $w W_{f}$ in terms of descent monomials.

Proposition 5.4 ([2, Proposition 3.7]) For any $w \in W_{e}^{+}$such that $w$ is maximal in $w W_{f}$, there is a unique expression for $w$ of the form

$$
w=u \cdot w_{0} y^{\lambda},
$$

where $u \in D^{S}$ and $\lambda \in Y_{+}^{+}$.

The next theorem simplifying the canonical basis of $\widehat{\mathscr{H}}^{+}$is a special case of a result of Xi ([17, Corollary 2.11]), also found independently by the author. We state here a combination of Lusztig's theorem (Theorem 5.1) and Xi's theorem.

Theorem 5.5 For $w=u \cdot w_{0} y^{\lambda}$ as in Proposition 5.4 we have the factorization

$$
C_{w}^{\prime}=s_{\lambda}(Y) C_{u w_{0}}^{\prime} .
$$

Remark 5.6 The general version of this theorem holds for the entire lowest two-sided cell of $W_{e}$ and in arbitrary type. The general version for type $A$ is used in the full version of this paper [1] to prove analogues of the results below for $\widehat{\mathscr{H}}^{+}$, rather than just $\widehat{\mathscr{H}}^{+} e^{+}$.

Let $e^{+}=C_{w_{0}}^{\prime}$. Then $A e^{+}$is the one-dimensional trivial left-module of $\mathscr{H}$ in which the $T_{i}$ act by $u$ for $i \in[n-1]$. The $\widehat{\mathscr{H}}^{+}$-module $\widehat{\mathscr{H}}^{+} e^{+}=\widehat{\mathscr{H}}^{+} \otimes \mathscr{H}^{+} e^{+}$is a $u$-analogue of the polynomial ring $R$. It can be identified with the cellular submodule of $\widehat{\mathscr{H}}^{+}$spanned by $\left\{C_{w}^{\prime}: w\right.$ maximal in $\left.w W_{f}\right\}$.

Let $\mathscr{R}$ denote the subalgebra of $\widehat{\mathscr{H}}^{+}$generated by the Bernstein generators $Y_{i}$. It is known that $\mathscr{R} \cong R$ as algebras. Write $\left(Y^{+}\right)_{\geq d}^{W_{f}} \subseteq \mathscr{R}$ for the set of $W_{f}$-invariant polynomials of degree at least $d$. Now Theorem 5.5 applied to the canonical basis of $\widehat{\mathscr{H}}^{+} e^{+}$yields the following corollary, which gives a $u$ analogue of the ring of coinvariants.

Corollary 5.7 The $\widehat{\mathscr{H}}_{n}^{+}$-module $\widehat{\mathscr{H}}_{n}^{+} e^{+}$has a cellular quotient equal to

$$
\mathscr{R}_{1^{n}}:=\widehat{\mathscr{H}}_{n}^{+} e^{+} / \widehat{\mathscr{H}}_{n}^{+}\left(Y^{+}\right)_{\geq 1}^{\mathcal{S}_{n}} e^{+}
$$

with canonical basis $\left\{C_{w}^{\prime}: w \in D^{S} w_{0}\right\}$. 


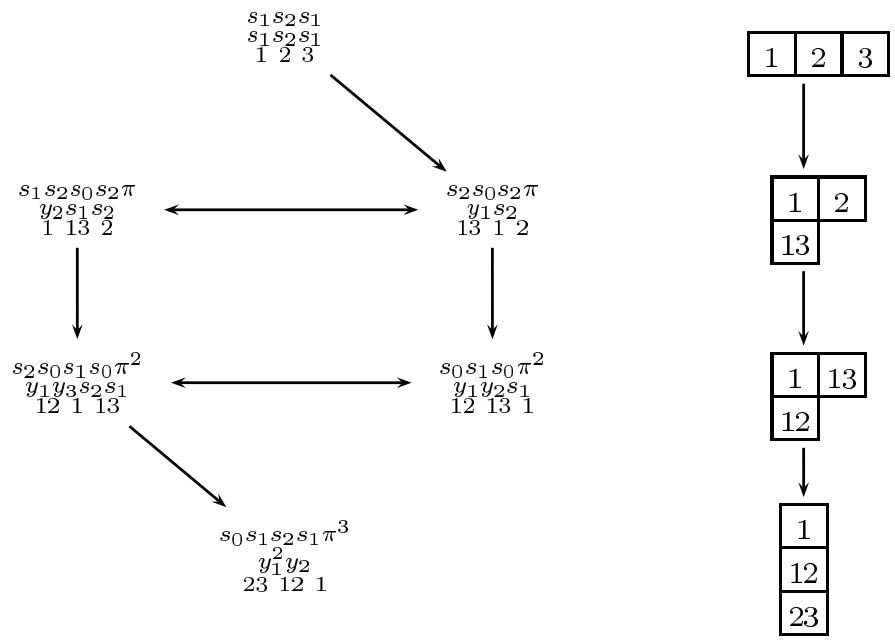

Fig. 1: On the left is the $W_{e}^{+}$-graph of $\mathscr{R}_{13}$ with three labels for each canonical basis element. The bottom labels are affine words. On the right are the corresponding left cells and the partial order $\leq \mathscr{R}_{1^{3}}$ on left cells.

Example 5.8 The $W_{e}^{+}$-graph $\mathscr{R}_{1^{3}}$ is drawn in Figure 1 . Arrows indicate relations in the preorder $\leq \mathscr{R}_{1^{n}}$ and those with a downward component are exactly the corotation-edges. Figure 2 depicts the left cells of the $W_{e}^{+}$-graph on $\mathscr{R}_{1^{5}}$ and the partial order $\leq \mathscr{R}_{1^{5}}$ on left cells.

These examples and the next proposition show that the partial order $\leq_{\mathscr{R}_{1}{ }^{n}}$ contains strictly more information than the cocyclage poset on SYT.

Let $\operatorname{CCP}\left(\mathscr{R}_{1^{n}}\right)$ be the subposet of $\operatorname{CCP}(P A T)$ on the set of tableaux corresponding to the cells of $\mathscr{R}_{1^{n}}$ and let $T+T^{\prime}$ denote the entry-wise sum of two tableau $T, T^{\prime}$ of the same shape. Using Proposition 5.2. we deduce the following

Proposition 5.9 The map $\mathrm{CCP}(S Y T) \rightarrow \mathrm{CCP}\left(\mathscr{R}_{1^{n}}\right), T \mapsto n T^{c c}+T$ is a color-preserving isomorphism of cocyclage posets.

For a PAT $P$ labeling a cell of $\mathscr{R}_{1^{n}}$, let ctype $(P)$ be ctype $(T)$, where $T$ is the SYT corresponding to $P$ in the bijection above.

\section{A $W_{e}^{+}$-graph version of the Garsia-Procesi modules}

The Garsia-Procesi approach to understanding the $R_{\lambda}=R / I_{\lambda}$ realizes $I_{\lambda}$ as the ideal of leading forms of functions vanishing on an orbit $\mathcal{S}_{n} \mathbf{a}$, for certain $\mathbf{a} \in \mathbb{C}^{n}=\operatorname{Spec} R$. We adapt this approach to the Hecke algebra setting using certain representations of $\widehat{\mathscr{H}}$ studied by Bernstein and Zelevinsky in order to prove our main result, Theorem 6.6, which shows that the $u$-analogues $\mathscr{R}_{\lambda}$ of the $R_{\lambda}$ are actually cellular.

Let $\mathcal{C}_{n}^{\mathbb{Z}}\left(\right.$ resp. $\mathcal{C}_{n}^{+\mathbb{Z}}$ ) be the category of finite-dimensional $\widehat{\mathscr{H}}_{n}$-modules (resp. $\widehat{\mathscr{H}}_{n}^{+}$-modules) in which the $Y_{i}$ 's have their eigenvalues in $u^{2 \mathbb{Z}}$. See [16] for many of the known results about the category $\mathcal{C}_{n}^{\mathbb{Z}}$. 


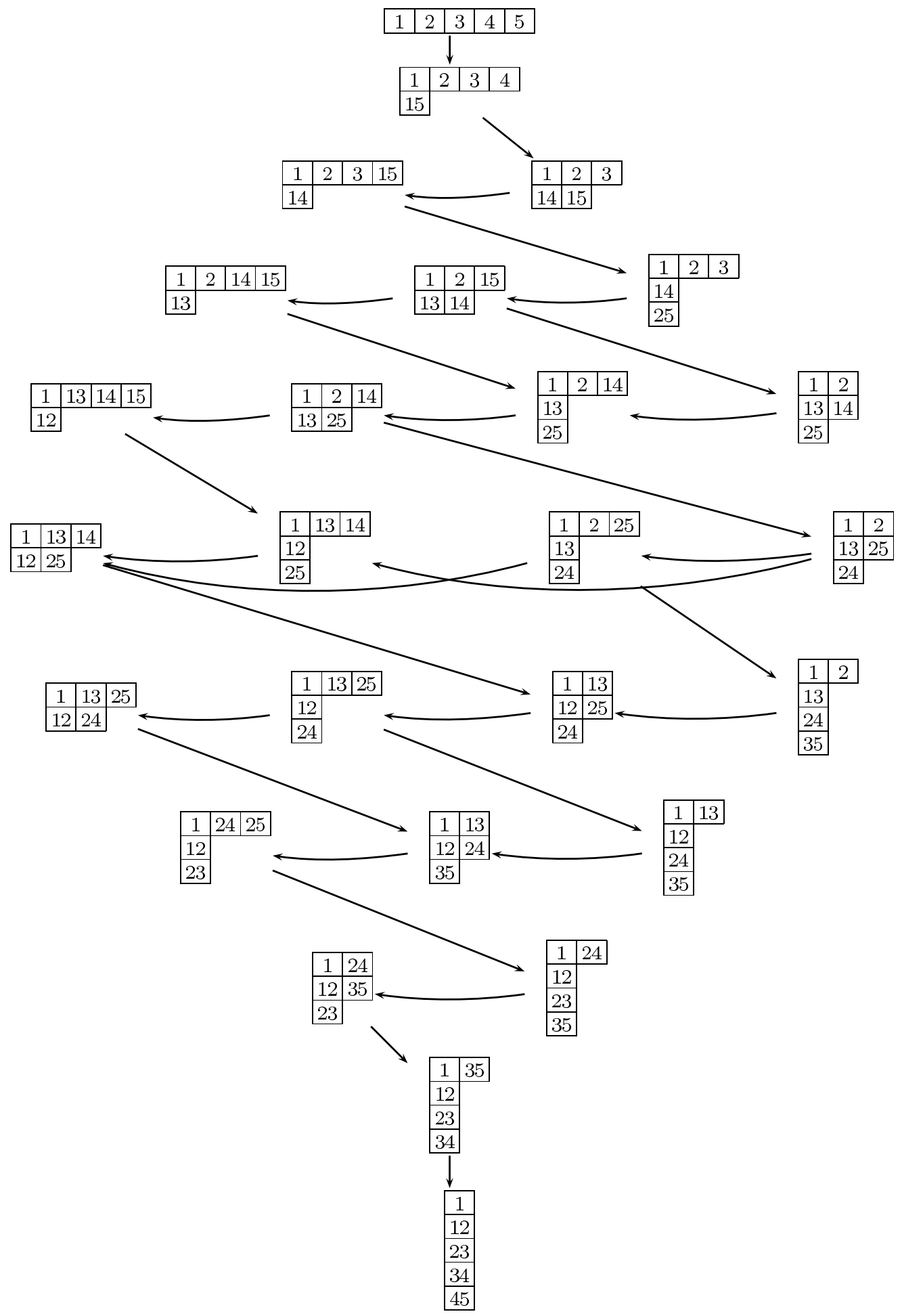

Fig. 2: The cells of the $W_{e}^{+}$-graph on $\mathscr{R}_{1^{5}}$. Edges are the covering relations of the partial order on cells. 
For $\eta=\left(\eta_{1}, \eta_{2}, \ldots, \eta_{r}\right)$ an $r$-composition of $n$, write $l_{j}=\sum_{i=1}^{j-1} \eta_{i}, j \in[r+1]$ for the partial sums of $\eta$ (where the empty sum is defined to be 0 ). Let $B_{j}$ be the interval $\left[l_{j}+1, l_{j+1}\right], j \in[r]$, and define

$$
J_{\eta}=\left\{s_{i}:\{i, i+1\} \subseteq B_{j} \text { for some } j\right\}
$$

so that $\mathcal{S}_{n J_{\eta}} \cong \mathcal{S}_{\eta_{1}} \times \cdots \times \mathcal{S}_{\eta_{r}}$.

Let $\widehat{\mathscr{H}}_{\eta}^{+} \cong \widehat{\mathscr{H}}_{\eta_{1}}^{+} \times \cdots \times \widehat{\mathscr{H}}_{\eta_{r}}^{+}$be the subalgebra of $\widehat{\mathscr{H}}^{+}$generated by $\mathscr{H}_{J_{\eta}}$ and $Y_{i}, i \in[n]$. For $\mathbf{a}=\left(a_{1}, \ldots, a_{r}\right) \in \mathbb{Z}^{r}$, let $\mathbf{C}_{\eta, \mathbf{a}}$ be the 1-dimensional representation of $\widehat{\mathscr{H}}_{\eta}^{+}$on which $\mathscr{H}_{J_{\eta}} \subseteq \widehat{\mathscr{H}}_{\eta}^{+}$acts trivially $\left(T_{i}\right.$ acts by $u$ for $s_{i} \in J_{\eta}$ ) and $Y_{l_{i}+1}$ acts by $u^{2 a_{i}}, i \in[r]$. The relations in $\widehat{\mathscr{H}}_{\eta}^{+}$demand that $Y_{l_{i}+k}$ acts by $u^{2\left(a_{i}-k+1\right)}$ for $l_{i}+k \in B_{i}$.

Next define $M_{\eta, \text { a }}$ to be the induced module

$$
M_{\eta, \mathbf{a}}=\widehat{\mathscr{H}}_{n}^{+} \otimes \widehat{\mathscr{H}}_{\eta}^{+} \mathbf{C}_{\eta, \mathbf{a}}
$$

For $M$ in $\mathcal{C}_{n}^{+\mathbb{Z}}$, the points of $M$ are the joint generalized eigenspaces for the action of the $Y_{i}$. The coordinates of a point $v$ of $M$ is the tuple $\left(c_{1}, \ldots, c_{n}\right)$ of generalized eigenvalues, also identified with the word $c_{1} c_{2} \cdots c_{n}$. The next proposition follows from a special case of well-known results about $\mathcal{C}_{n}^{\mathbb{Z}}$.

Proposition 6.1 If the intervals $\left[a_{i}-\eta_{i}, a_{i}\right]$ are disjoint, then the points of $M_{\eta, a}$ are 1-dimensional and are the shuffles of the words

$$
u^{2 a_{1}} u^{2\left(a_{1}-1\right)} \cdots u^{2\left(a_{1}-\eta_{1}\right)}, u^{2 a_{2}} \cdots u^{2\left(a_{2}-\eta_{2}\right)}, \ldots, u^{2 a_{r}} u^{2\left(a_{r}-1\right)} \cdots u^{2\left(a_{r}-\eta_{r}\right)} .
$$

An essential part of the Garsia-Procesi approach is that the ideal of leading forms of functions vanishing on $\mathcal{S}_{n}$ a affords the same $\mathcal{S}_{n}$-representation as the ideal of functions vanishing on $\mathcal{S}_{n} \mathbf{a}$. The analogous fact in this setting is

Proposition 6.2 Let $M_{\eta, a}$ be as above. If $M_{\eta, a}$ is irreducible, then it contains an element $v^{+}$such that, setting $N=\operatorname{Ann} v^{+}, \widehat{\mathscr{H}}^{+} e^{+} / N e^{+} \cong M_{\eta, a}$ as $\widehat{\mathscr{H}}^{+}-$modules. It follows that $\widehat{\mathscr{H}}^{+} e^{+} / \operatorname{gr}(N) e^{+} \cong$ $\widehat{\mathscr{H}}^{+} e^{+} / N e^{+} \cong M_{\eta, \boldsymbol{a}}$ as $\mathscr{H}$-modules.

The ideals $I_{\lambda}$ are generated by certain elementary symmetric functions in subsets variables, also known as Tanisaki generators (see [5, 6]). By the next theorem, certain $C_{w}^{\prime} \in \mathscr{R}_{1^{n}}$ are essentially these generators. This will relate the ideals $\operatorname{gr}\left(\operatorname{Ann} M_{\eta, \mathbf{a}}\right) e^{+}$to the canonical basis of $\widehat{\mathscr{H}}^{+} e^{+}$.

Theorem 6.3 For $k, d \in[n]$ such that $d \leq k$, let $w$ be the maximal element of $y_{k-d+1} y_{k-d+2} \ldots y_{k} W_{f}$. Then

$$
C_{w}^{\prime}=u^{d(k-n)} s_{1^{d}}\left(Y_{1}, \ldots, Y_{k}\right) C_{w_{0}}^{\prime} .
$$

Suppose $d, k \in[n], d \leq k$. Consider the following property of a partition $\lambda \vdash n$ :

$$
d>k-n+\lambda_{1}^{\prime}+\cdots+\lambda_{n-k}^{\prime}
$$

where $\lambda^{\prime}$ is the partition conjugate to $\lambda$.

A result of Garsia-Procesi ([5, Proposition 3.1]) carries over to this setting virtually unchanged. For a composition $\eta$, let $\eta_{+}$denote the partition obtained from $\eta$ by sorting its parts in decreasing order. 
Proposition 6.4 Suppose $\eta$ is an $r$-composition of $n$ with $\lambda:=\eta_{+}$, and $k, d \in[n], d \leq k$, such that (11) holds. If $M_{\eta, a}$ satisfies the hypotheses of Proposition 6.1, then

$$
s_{1^{d}}\left(Y_{1}, \ldots, Y_{k}\right) \in \operatorname{gr}\left(\operatorname{Ann} M_{\eta, \boldsymbol{a}}\right) .
$$

For $h \in \widehat{\mathscr{H}}^{+}$, write $\left[C_{w}^{\prime}\right] h$ for the coefficient of $C_{w}^{\prime}$ of $h$ written as an $A$-linear combination of $\left\{C_{w}^{\prime}\right.$ : $\left.w \in W_{e}^{+}\right\}$. Define $\langle,\rangle_{\lambda}: \mathscr{\mathscr { H }}^{+} \times \widehat{\mathscr{H}}^{+} e^{+} \rightarrow A$ by

$$
\left\langle h_{1}, h_{2}\right\rangle_{\lambda}=\left[C_{g_{\lambda}}^{\prime}\right] h_{1} h_{2},
$$

where $g_{\lambda}$ is the row reading word of $G_{\lambda}$.

Through the work of Kazhdan-Lusztig and Beilinson-Bernstein-Deligne-Gabber we know (see, for instance, [13]) that the structure coefficients of the $C^{\prime}$ 's are nonnegative. Using this, we prove

Corollary 6.5 If $\gamma \in \mathcal{I}_{\lambda}^{\text {pair }}, \gamma \in \Gamma_{W_{e}^{+}}$, then $\delta \leq \widehat{\mathscr{H}}^{+} \gamma\left(\delta \in \Gamma_{W_{e}^{+}}\right)$implies $\delta \in \mathcal{I}_{\lambda}^{\text {pair }}$, i.e., the cellular submodule generated by $\gamma$ is contained in $\mathcal{I}_{\lambda}^{\text {pair }}$.

We now come to our main result.

Theorem 6.6 Suppose $M_{\eta, a}$ satisfies the hypotheses of Propositions 6.1] and 6.2 and maintain the notation of Proposition 6.2. Then the following submodules of $\widehat{\mathscr{H}}^{+} e^{+}$are equal.

(i) $\mathcal{I}_{\lambda}^{o}:=\operatorname{gr}\left(\operatorname{Ann} v^{+}\right) e^{+}$,

(ii) $\mathcal{I}_{\lambda}^{T}:=\widehat{\mathscr{H}}^{+}\left\{s_{1^{d}}\left(Y_{1}, \ldots, Y_{k}\right): d, k, \lambda\right.$ satisfy $\left.(11)\right\} e^{+}$,

(iii) $\mathcal{I}_{\lambda}^{\text {pair }}:=\left\{v \in \widehat{\mathscr{H}}^{+} e^{+}:\left\langle\widehat{\mathscr{H}}^{+}, v\right\rangle_{\lambda}=0\right\}$,

(iv) $\mathcal{I}_{\lambda}^{\text {cell }}:=$ The maximal cellular submodule of $\widehat{\mathscr{H}}^{+} e^{+}$not containing $\Gamma_{G_{\lambda}}$,

(v) $\mathcal{I}_{\lambda}^{\text {cat }}:=A\left\{C_{w}^{\prime}: \operatorname{ctype}(P(w)) \unrhd \lambda\right\}$.

The abbreviations o, T, pair, are shorthand for orbit, Tanisaki, and pairing. Also note that modules $M_{\eta, \mathbf{a}}$ satisfying the hypotheses of Propositions 6.1 and 6.2 exist by the general theory. For instance, if $\left|a_{i}-a_{j}\right|>>0$ for all $i \neq j$, then these hypotheses are satisfied.

Proof sketch: The inclusion $\mathcal{I}_{\lambda}^{\mathrm{T}} \subseteq \mathcal{I}_{\lambda}^{\mathrm{o}}$ follows from Proposition 6.4. An argument of a similar flavor to Proposition 6.4 together with Proposition 6.2 yields $\mathcal{I}_{\lambda}^{\mathrm{o}} \subseteq \mathcal{I}_{\lambda}^{\text {pair }}$. Next, the inclusion $\mathcal{I}_{\lambda}^{\mathrm{T}} \subseteq \mathcal{I}_{\lambda}^{\text {pair }}$ together with Theorem 6.3 and Corollary 6.5 show that $\mathcal{I}_{\lambda}^{\mathrm{T}}$ is cellular, implying $\mathcal{I}_{\lambda}^{\mathrm{T}} \subseteq \mathcal{I}_{\lambda}^{\text {cell }}$. It follows from the catabolism insertion algorithm (Algorithm 4.1 for any $w$ satisfying ctype $(P(w)) \unrhd \lambda$, there is a sequence of ascent-edges and corotation-edges from $w$ to $g_{\lambda}$. This proves $\mathcal{I}_{\lambda}^{\text {cell }} \subseteq \mathcal{I}_{\lambda}^{\text {cat }}$. Finally, a dimension counting argument using the $u=1$ results of Garsia-Procesi and Bergeron-Garsia (see [6]) and the standardization map of Lascoux (see [15]) completes the proof.

Given the theorem, define $\mathscr{R}_{\lambda}$ to be $\widehat{\mathscr{H}}^{+} e^{+} / \mathcal{I}_{\lambda}$ for $\mathcal{I}_{\lambda}$ equal to any (all) of the submodules above. By description (iv), $\mathscr{R}_{\lambda}$ is the minimal cellular quotient of $\widehat{\mathscr{H}}^{+} e^{+}$containing $\Gamma_{G_{\lambda}}$. By description (iii) and the description of $R_{\lambda}$ from the introduction, $\mathscr{R}_{\lambda}$ is a $u$-analogue of $R_{\lambda}$.

\section{Acknowledgements}

I am deeply grateful to my advisor Mark Haiman for his wonderful ideas, his generous advice, and our many detailed discussions that made this paper possible. I am also grateful to John Wood for help typing and typesetting figures. 


\section{References}

[1] Jonah Blasiak. Cyclage, catabolism, and the affine Hecke algebra. In preparation, 2009.

[2] Jonah Blasiak. A factorization theorem for affine Kazhdan-Lusztig basis elements. Preprint, arXiv:0908.0340v1, 2009.

[3] Jonah Blasiak. An insertion algorithm for catabolizability. Preprint, arXiv:0908.1967v1, 2009.

[4] Li-Chung Chen. Private communication.

[5] A. M. Garsia and C. Procesi. On certain graded $S_{n}$-modules and the $q$-Kostka polynomials. Adv. Math., 94(1):82-138, 1992.

[6] Mark Haiman. Combinatorics, symmetric functions, and Hilbert schemes. In Current developments in mathematics, 2002, pages 39-111. Int. Press, Somerville, MA, 2003.

[7] Mark Haiman. Cherednik algebras, Macdonald polynomials and combinatorics. In International Congress of Mathematicians. Vol. III, pages 843-872. Eur. Math. Soc., Zürich, 2006.

[8] David Kazhdan and George Lusztig. Representations of Coxeter groups and Hecke algebras. Invent. Math., 53(2):165-184, 1979.

[9] L. Lapointe, A. Lascoux, and J. Morse. Tableau atoms and a new Macdonald positivity conjecture. Duke Math. J., 116(1):103-146, 2003.

[10] Alain Lascoux. Cyclic permutations on words, tableaux and harmonic polynomials. In Proceedings of the Hyderabad Conference on Algebraic Groups (Hyderabad, 1989), pages 323-347, Madras, 1991. Manoj Prakashan.

[11] Alain Lascoux and Marcel-P. Schützenberger. Le monoïde plaxique. In Noncommutative structures in algebra and geometric combinatorics (Naples, 1978), volume 109 of Quad. "Ricerca Sci.", pages 129-156. CNR, Rome, 1981.

[12] George Lusztig. Singularities, character formulas, and a $q$-analog of weight multiplicities. In Analysis and topology on singular spaces, II, III (Luminy, 1981), volume 101 of Astérisque, pages 208229. Soc. Math. France, Paris, 1983.

[13] George Lusztig. Cells in affine Weyl groups. In Algebraic groups and related topics (Kyoto/Nagoya, 1983), volume 6 of Adv. Stud. Pure Math., pages 255-287. North-Holland, Amsterdam, 1985.

[14] Yuval Roichman. Induction and restriction of Kazhdan-Lusztig cells. Adv. Math., 134(2):384-398, 1998.

[15] Mark Shimozono and Jerzy Weyman. Graded characters of modules supported in the closure of a nilpotent conjugacy class. European J. Combin., 21(2):257-288, 2000.

[16] M. Vazirani. Parameterizing Hecke algebra modules: Bernstein-Zelevinsky multisegments, Kleshchev multipartitions, and crystal graphs. Transform. Groups, 7(3):267-303, 2002.

[17] Nan Hua Xi. The based ring of the lowest two-sided cell of an affine Weyl group. J. Algebra, 134(2):356-368, 1990. 